\title{
Energy Landscapes Reveal Agonist Control of G Protein-Coupled Receptor Activation via Microswitches
}

\author{
Oliver Fleetwood, Pierre Matricon, Jens Carlsson, and Lucie Delemotte* \\ Cite This: Biochemistry 2020, 59, 880-891 \\ Read Online
}

ABSTRACT: Agonist binding to $G$ protein-coupled receptors (GPCRs) leads to conformational changes in the transmembrane region that activate cytosolic signaling pathways. Although highresolution structures of different receptor states are available, atomistic details of allosteric signaling across the membrane remain elusive. We calculated free energy landscapes of $\beta_{2}$ adrenergic receptor activation using atomistic molecular dynamics simulations in an optimized string of swarms framework, which shed new light on how microswitches govern the equilibrium between conforma-

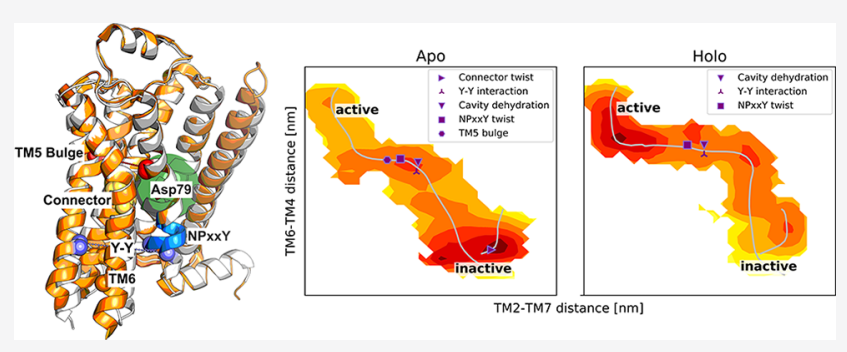
tional states. Contraction of the extracellular binding site in the presence of the agonist BI-167107 is obligatorily coupled to conformational changes in a connector motif located in the core of the transmembrane region. The connector is probabilistically coupled to the conformation of the intracellular region. An active connector promotes desolvation of a buried cavity, a twist of the conserved NPxxY motif, and an interaction between two conserved tyrosines in transmembrane helices 5 and 7 (Y-Y motif), which lead to a larger population of active-like states at the $\mathrm{G}$ protein binding site. This coupling is augmented by protonation of the strongly conserved Asp $79^{2.50}$. The agonist binding site hence communicates with the intracellular region via a cascade of locally connected microswitches. Characterization of these can be used to understand how ligands stabilize distinct receptor states and contribute to development drugs with specific signaling properties. The developed simulation protocol can likely be transferred to other class A GPCRs.

G protein-coupled receptors (GPCRs) are membrane proteins that activate cellular signaling pathways in response to extracellular stimuli. There are more than 800 GPCRs in the human genome, ${ }^{1}$ and these recognize a remarkably large repertoire of ligands such as neurotransmitters, peptides, proteins, and lipids. This large superfamily plays essential roles in numerous physiological processes and has become the most important class of drug targets. ${ }^{2}$ All GPCRs share a common architecture of seven transmembrane (TM) helices, which recognizes the cognate ligand in the extracellular region and triggers intracellular signals via a more conserved cytosolic domain (Figure 1). ${ }^{3,4}$ GPCRs are inherently flexible proteins that exist in multiple conformational states, and drug binding alters the relative populations of these. Agonists will shift the equilibrium toward active-like receptor conformations, which promote binding of $\mathrm{G}$ proteins leading to initiation of signaling via multiple pathways. In the apo state, GPCRs can still access active-like conformations and thereby exhibit a smaller degree of signaling, which is termed basal activity.

Over the past decade, breakthroughs in GPCR structure determination have provided insights into the process of activation at atomic resolution (Figure 1 ). In particular, crystal structures of the $\beta_{2}$ adrenergic receptor $\left(\beta_{2} \mathrm{AR}\right)$ in both active and inactive conformational states ${ }^{5-14}$ have revealed hallmarks of GPCR activation. The observations made for this prototypical receptor have recently been reinforced by crystal and cryogenic electron microscopy (cryo-EM) structures of other family members. ${ }^{15}$ The most prominent features of GPCR activation are a large outward movement of TM6 $(1.0-1.4 \mathrm{~nm})$ and a slight inward shift of TM7 on the intracellular side (Figure 1), which create a cavity for binding of cytosolic proteins.

Conserved changes in the extracellular part are more difficult to discern due to the lower degree of sequence conservation in this region. In general, the structural changes are relatively subtle and involve only a small contraction of the orthosteric site. ${ }^{5-7}$ In the case of the $\beta_{2} \mathrm{AR}$, the catechol group of adrenaline forms hydrogen bonds with Ser203 5.42 and Ser207 $7^{5.46}$ (superscripts denote Ballesteros-Weinstein numbering ${ }^{16}$ ), which leads to an $\sim 0.2 \mathrm{~nm}$ inward bulge of TM5. These structural changes then propagate through the receptor via several conserved motifs. The rearrangement of TM5 influences a connector region $\left(\mathrm{P}^{5.50} \mathrm{I}^{3.40} \mathrm{~F}^{6.44}\right.$ motif), which is in contact with the highly conserved Asp79 2.50 and $\mathrm{N}^{7.49} \mathrm{P}^{7.50} \mathrm{xxY}^{7.53}$ motif via a network of ordered water

Received: September 12, 2019

Revised: January 30, 2020

Published: January 30, 2020 


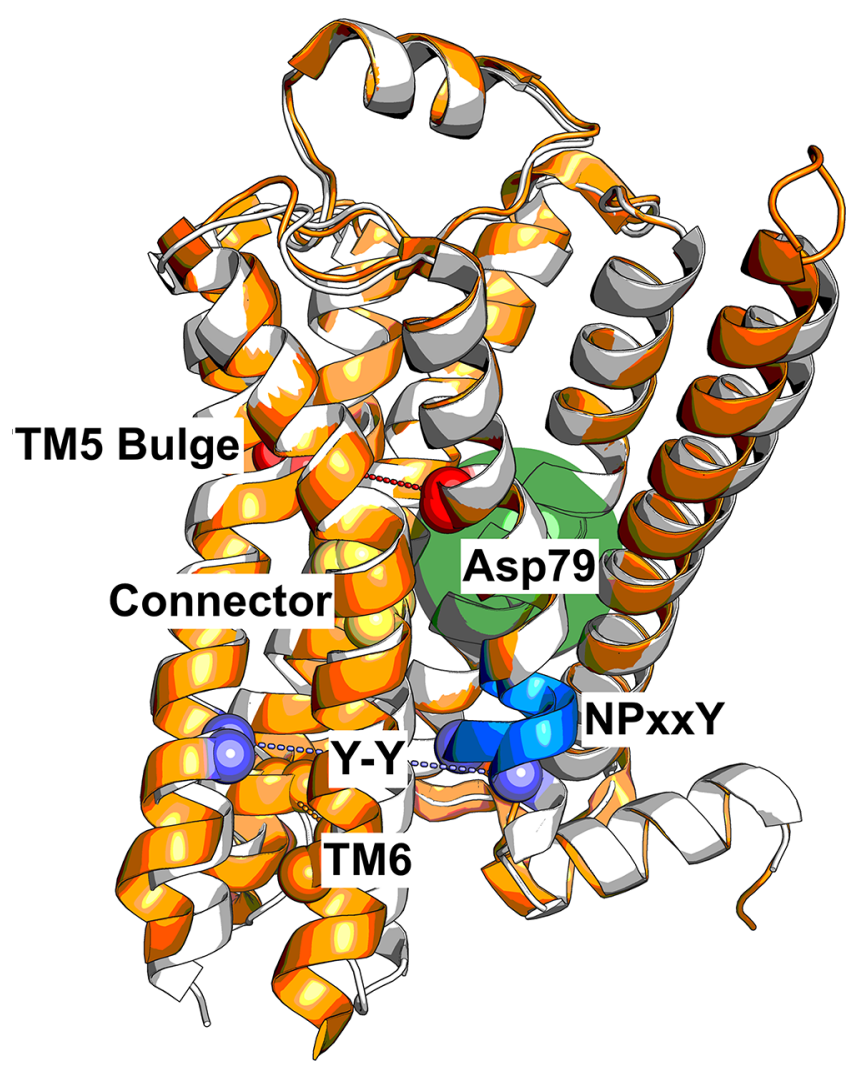

Figure 1. Activation mechanism of GPCRs involving a series of microswitches. Binding of the agonist BI-167107 leads to an inward bulge of TM5 (quantified as the distance between Ser207 $7^{5.46}$ and Gly315 $15^{7.41}$, red spheres), which leads to a conformational change in the connector region (Ile $121^{3.40}$ and Phe $282^{6.44}$, yellow spheres). The transmembrane cavity surrounding Asp $79^{2.50}$ is dehydrated (filled green circle), and the NPxxY motif (blue cartoon) twists upon activation, leading to the $\mathrm{Y}-\mathrm{Y}$ interaction (Tyr326 $6^{7.53}$ and Tyr219.58, purple spheres). TM6 moves outward to create the $\mathrm{G}$ protein binding site. The inactive (PDB entry $2 \mathrm{RH}^{5}$ ) and active (PDB entry $3 \mathrm{POG}^{6}$ ) structures are colored white and orange, respectively.

molecules. A water-filled cavity surrounding Asp79 2.50 contributes to the stabilization of a sodium ion in several crystal structures of the inactive state, e.g., for the $\beta_{1} \mathrm{AR} .{ }^{17,18} \mathrm{Upon}$ activation, the cavity collapses, leading to dehydration, displacement of the sodium ion and potentially protonation of Asp7 $9^{2.50} .^{19,20}$ Activation also involves a twist of the NPxxY motif, which reorients $\mathrm{Tyr} 326^{7.53}$ to a position where it can form a water-mediated interaction with Tyr2195.58 (Y-Y interaction $)^{21}$ and together with the displacement of TM6 enable formation of the $G$ protein binding site. Characterization of the role these individual microswitches play in determining the conformational ensemble of structures could guide the design of drugs with tailored signaling profiles.

The allosteric control of GPCR activation by extracellular ligands cannot be fully understood from the static structures captured by crystallography or cryo-EM. Mutagenesis and spectroscopy studies ${ }^{22-25}$ have suggested that the efficacy of a ligand is determined by a complex interplay between different microswitches and that population of distinct states leads to specific functional outcomes. Molecular dynamics (MD) simulations are well suited to study the conformational landscape of GPCRs as this method can provide an atomistic view of the flexible receptor in the presence of a membrane, an aqueous solvent, and ligands. ${ }^{26-34}$ The seminal paper by Dror et al. ${ }^{31}$ provided insights into the activation mechanism of the $\beta_{2} \mathrm{AR}$ by monitoring how a crystallized active conformation relaxed to an inactive state upon removal of the intracellular binding partner, a $\mathrm{G}$ protein mimicking nanobody, using $\mathrm{MD}$ simulations. Although key conformational changes involved in the transition from active to inactive conformations were identified in these simulations, this approach has inherent limitations. Indeed, understanding the roles of different microswitches in activation and the strength of the coupling between these requires mapping of the relevant free energy landscapes, which are still too costly to calculate using bruteforce MD simulations. Enhanced sampling methods, on the other hand, provide a means to explore the conformational landscapes of proteins at a relatively low computational $\operatorname{cost}^{35}$ and have been exploited to study the activation mechanism of GPCRs. ${ }^{26-28,30,32-34}$

In this work, we aimed to identify the most probable path describing the transition between inactive and active states of $\beta_{2} \mathrm{AR}$ and to characterize the full conformational ensemble along this pathway at an atomistic level of resolution. A new version of the string method with swarms of trajectories was first developed for this purpose. The free energy landscapes associated with $\beta_{2} \mathrm{AR}$ activation revealed that whereas agonist binding is only loosely connected to outward movement of TM6, the coupling between microswitch pairs in the transmembrane region ranges from weak to strong. We observed sodium interacting with several binding sites in a state-dependent manner that was consistent with experimental data. Our approach also allowed us to investigate the influence of Asp $79^{2.50}$ protonation and sodium ions on the energy landscape. A protonated (neutral) Asp $79^{2.50}$ favored active-like conformations and led to a distinct conformational state of the NPxxY motif, which closely resembles agonist-bound and active structures of other class A GPCRs. Finally, we demonstrated that the collective variables $(\mathrm{CVs})$ used in this study discriminate active from inactive class A GPCR structures in general. By using the activation path of $\beta_{2} \mathrm{AR}$ as input to simulations of other receptors, our approach can therefore likely be transferred to study activation of other class A GPCRs at a modest computational cost.

\section{METHODS}

All swarms of trajectories simulations were initiated with the coordinates from the crystal structure of the active $\beta_{2} \mathrm{AR}$ solved in complex with the agonist BI-167107 (hereafter termed the agonist) and an intracellular nanobody (PDB entry 3P0G) (the first two simulation systems in Table S1). The Asn187Glu mutation in 3 P0G was reverted, and Glu122 $2^{3.41}$ was protonated due to its localization in a hydrophobic pocket, as has been common practice in other simulations of this receptor. ${ }^{36}$ Residues His $172^{4.64}$ and His $178^{4.70}$ were both protonated at the $\varepsilon$ position. The systems were parametrized using the CHARMM $36 \mathrm{~m}$ force field ${ }^{37}$ and the TIP3P water model. ${ }^{38}$ The protein was inserted in a POPC $^{39}$ bilayer and solvated in explicit solvent. $\mathrm{Na}^{+}$and $\mathrm{Cl}^{-}$ions were added at a concentration of $0.15 \mathrm{M}$. System preparation was performed using CHARMM-GUI. ${ }^{40}$ MD simulations were run with GROMACS $2016.5^{41}$ patched with plumed $2.4 .1^{42}$ under a 1 atm pressure and at $310.15 \mathrm{~K}$.

To identify CVs, we performed clustering ${ }^{43}$ on the frames from an unrestrained $\mathrm{MD}$ simulation trajectory of $\beta_{2} \mathrm{AR}$ (condition $\mathrm{A}$ in Dror et al. ${ }^{31}$ ) (Figure S1). The CVs were selected by training a multilayer perceptron classifier ${ }^{44}$ using as 
input all the inter-residue distances and as output the cluster ID, followed by using Deep Taylor decomposition ${ }^{45}$ to find key distances that could discriminate between clusters.

The end points of the main strings describing the transition between inactive and active states (subscript $t$ ) were fixed to the output coordinates of equilibrated structures of each state (PDB entries $2 \mathrm{RH}^{5}$ and $3 \mathrm{POG},{ }^{6}$ respectively). The initial path for simulations of $\mathrm{Holo}_{t}$ was guessed using data from Dror et al.. A rough estimate of the free energy landscape was calculated from the probability density landscape estimated using the Scikit-learn ${ }^{44}$ kernel density estimator with automatic bandwidth detection (Figure S2). Two additional short strings were set up to increase the level of sampling in the active (subscript $a$ ) and inactive (subscript $i$ ) regions. The average, partially converged path between iterations 20 and 30 from $\mathrm{Holo}_{t}$ was used as the input path for simulations $\mathrm{Apo}_{t}$ and $\mathrm{Holo}_{A s h 79, t}$. All active $\left(\mathrm{Apo}_{a}, \mathrm{Holo}_{a}\right.$ and $\left.\mathrm{Holo}_{A s h 79, a}\right)$ and inactive $\left(\mathrm{Apo}_{i}, \mathrm{Holo}_{i}\right.$, and $\left.\mathrm{Holo}_{A s h 79, i}\right)$ substrings were initiated as straight paths between the end points. Even though the system was initiated from the nanobody-bound structure 3P0G, the active state substring sampled conformations closer to the G protein-bound structure 3SN6 (Figure S3). ${ }^{7}$ The swarms of trajectories simulations with optimizations (Supplementary Methods and Figures S4 and S5) were run for 300 iterations, at which point the strings had not changed on average for many iterations (Figures S6 and S7) and the posterior distribution of free energy profiles given the data was narrow (Figure S8).

By discretizing the system into microstates, or bins, we were able to use the short trajectories from the swarms to create a transition matrix and derive the free energy distribution of the system $^{46}$ along a given variable (Figure S9). In practice, the transition probabilities $T_{i j}$ of the transition matrix $\mathbf{T}$ can be estimated from the normalized number of transitions, $N_{i j}$, from bin $i$ to bin $j: T_{i j}=N_{i j} / \sum_{k} N_{i k}$. The transition matrix of a physical system at equilibrium is constrained by detailed balance, such that for the stationary probability distribution, $\rho$, $\rho_{i} T_{i j}=\rho_{j} T_{j i}$. A Metropolis Markov chain Monte Carlo (MCMC) method was used to sample over the posterior distribution of transition matrices, given the unregularized elements of $T_{i j}{ }^{47}$ and thereby obtain a distribution of free energy profiles for $\rho$ (Figures S8, S10, and S11). All code to run the simulations and reproduce the results in this paper is available for download. ${ }^{48}$

\section{RESULTS}

Optimizing the Enhanced Sampling Protocol for Increased Efficiency. We aimed to compute the most probable transition pathway linking the inactive and active states of $\beta_{2} \mathrm{AR}$ and the relative free energy of the states lining this pathway. For this purpose, we chose the string of swarms method. ${ }^{49}$ In this framework, the minimum free energy path in an $\mathrm{N}$-dimensional collective variable $(\mathrm{CV})$ space is estimated iteratively from the drift along the local gradient of the free energy landscape (Figure S5). From each point on the string, a number of short trajectories are launched (a swarm), which enables us to calculate the drift. The string is then updated considering this drift and reparametrized to ensure full sampling of the configurational space along the pathway. Convergence is reached when the string diffuses around an equilibrium position. The method allows sampling of a highdimensional space at a relatively inexpensive computational cost because it samples only along the one-dimensional path of interest.

We characterized the pathway linking equilibrated conformations originating from active and inactive structures of the $\beta_{2} \mathrm{AR}$ (PDB entries 3P0G and 2RH1, respectively), adding two short strings spanning the active and the inactive regions to increase sampling of the end state environments (see Methods and Supplementary Methods). First, we characterized a CV set that embeds receptor activation by automatically inferring the inter-residue distances that are related to activation. On the basis of one of Dror et al.'s simulation trajectories of spontaneous deactivation of the $\beta_{2} \mathrm{AR},{ }^{31}$ we identified metastable states by clustering simulation configurations, followed by classification of these by training a fully connected neural network to identify states. ${ }^{50}$ The most important input features for classification were identified via deep Taylor decomposition ${ }^{45}$ and taken as CVs (Figure 2 and Figure S1). The set of CVs we inferred was a network of interatomic distances among all seven TM helices (Figure 2). Encouragingly, this five-dimensional CV set captured the main degrees of freedom implicated in $\beta_{2} \mathrm{AR}$ activation, including the large outward movement of TM6 and a smaller inward shift of TM7 at the intracellular face of the receptor.

To accelerate convergence of the string optimization, we initiated our string simulation from a rough guess of the minimum free energy landscape. The latter was obtained by estimating the density of points from Dror et al.'s trajectory in this CV space (Figure S2). We also introduced algorithmic improvements to the string of swarms method. We adaptively chose the number of trajectories in a swarm, gradually increased the number of points on the string, and introduced a reparametrization algorithm that improves performance and promotes exchanges of configurations between adjacent string points (Supplementary Methods and Figures S4 and S5). We carried out 300 iterations of string optimization for each system, considering a number of points on the string ranging from 20 to 43 and swarms consisting of $16-3210$ ps trajectories. Because we sought to sample only the vicinity of the most probable activation path, derivation of a converged free energy landscape required a mere total of approximately 2-3 $\mu$ s of simulation time (Figures S6-S8, S10, and S11).

Minimum Free Energy Pathway of $\beta_{2} A R$ Activation. We derived the most probable transition path (Figure S6) between the active and inactive states of $\beta_{2} \mathrm{AR}$ in the absence and prescence of an agonist ligand. The swarm trajectories allowed us to compute transitions between discrete states in the vicinity of the most probable transition path (Supplementary Methods and Figure S9) and to derive the associated free energy landscape describing the outward movement of TM6 and the inward shift of TM7 upon activation (Figure 3).

For the apo receptor, one distinguishes three minima: one in the active region, one in the inactive one, and an intermediate one between these (Figure 3a). As anticipated, regions close to the inactive end point are stabilized relative to the other two states. Binding of an agonist changes the number of minima to four and shifts the relative stability of states, making regions close to the active conformation of lower free energy (Figure 3b). A number of characteristic variables (defined in Table S2-S3) were calculated for the last iteration of the swarm of trajectories simulations. By localizing sudden shifts in the values of these parameters, we could pinpoint the location of important events in activation on the string (Figure 3). In the absence of a bound agonist, the connector assumes an active 


\section{a}

Input:

interresidue

distances

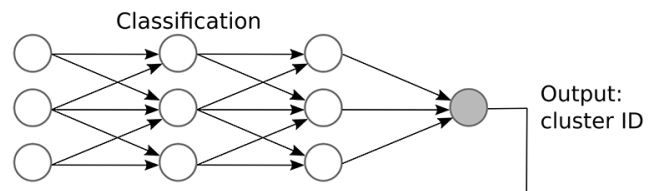

Key features: important interresidue distances
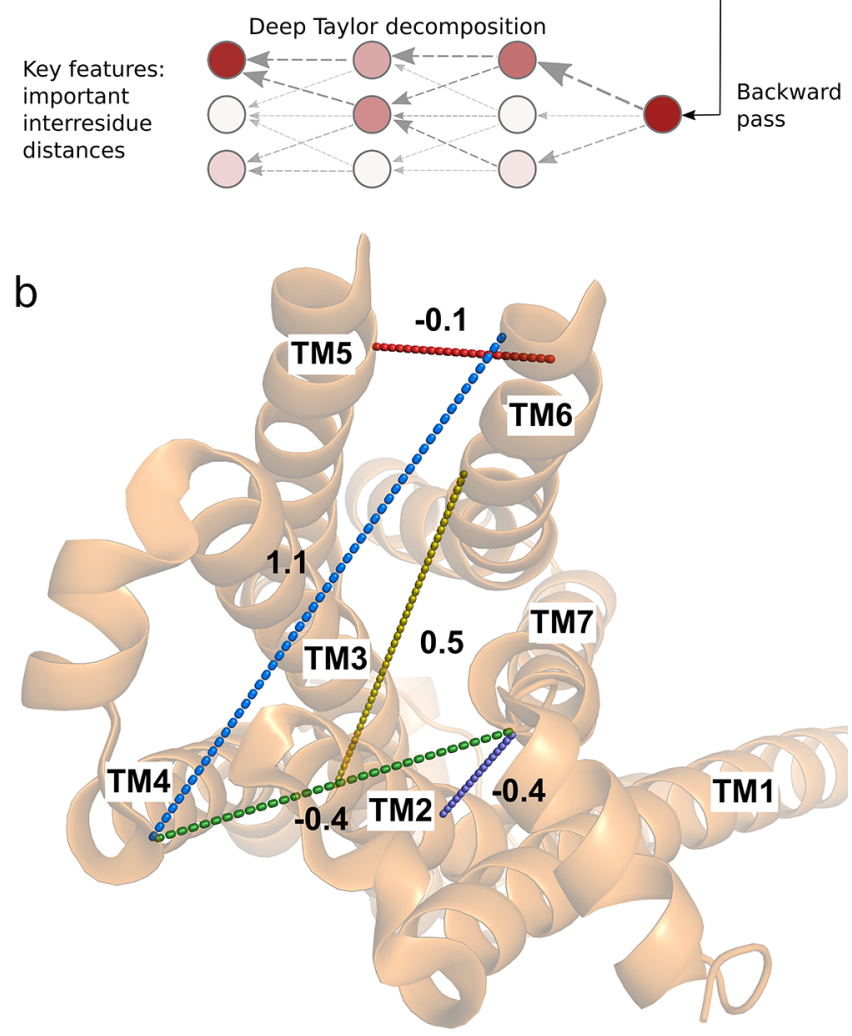

Figure 2. Five-dimensional collective variable (CV) space used to optimize the minimum free energy path identified in a data-driven manner. (a) A fully connected neural network was trained to classify configurations in active, intermediate, and inactive metastable states (clusters). Deep Taylor decomposition was then used to identify the most important input inter-residue distances for the classification decision. The top-ranked distances were used as CVs. (b) The five CVs used in this work projected onto an intracellular view of the active crystal structure (PDB entry $3 \mathrm{POG}$ ). The CVs corresponding to TM2-TM7, TM6-TM4, TM7-TM4, TM3-TM6, and TM6-TM5 distances defined in Table S2 are shown as purple, blue, green, yellow, and red dashed lines, respectively. The change of these distance CVs from the inactive to the active state structures is reported in nanometers.

conformation in the early stages of activation. The Asp79 2.50 cavity then dehydrates, followed by the formation of the $\mathrm{Y}-\mathrm{Y}$ interaction and NPxxY twist. Finally, the TM5 bulge forms before the fully active conformation is reached, confirming an allosteric communication between the orthosteric site and intracellular region.

Binding of the agonist ligand modifies the order in which the helices rearrange, as can be seen when projecting the minimum free energy path along various CVs (Figure 3 and Figure S6). The most probable activation pathway begins with an outward movement of TM6, followed by the inward shift of TM7. The connector and the bulge in TM5 are locked in their active-like states in the presence of the agonist, which enables large outward movements of TM6. As in the apo case, to reach the fully active conformation, the Asp79 ${ }^{2.50}$ cavity is dehydrated and the $\mathrm{Y}-\mathrm{Y}$ interaction forms shortly before the NPxxY twists. In the holo simulation, the binding mode of the ligand remained stable along the activation path (Figure S12a,b,k,1), and it maintained stable interactions with key residues Asp113 $3^{3.32}$, Ser203 $3^{5.43}$, Ser207 $7^{5.46}$, and Asn312 ${ }^{7.38}$ (Figure S12a-j). Occasionally, the shortest heavy atom distance from the ligand to Ser $203^{5.43}$ was almost $4 \AA$, although these fluctuations were independent of the receptor state (Figure S12e,f).

Coupling between Orthosteric and G Protein Binding Sites. As the final configurations of the string are at equilibrium in all dimensions, the trajectories from the last iterations can be used to compute the free energy landscape as a function of any variables (Supplementary Methods). This allowed a detailed analysis of how conformational changes induced by agonist binding propagate through the receptor to the $\mathrm{G}$ protein binding site. The roles of conserved microswitches were assessed by comparing free energy landscapes in the absence and presence of a bound agonist. We first evaluated how the TM5 bulge, which reflects how the binding site contracts upon activation, influences the intracellular distance between TM6 and TM3 (Figure 4a,b). In the absence of a bound agonist, the receptor accessed both active and inactive conformations of the binding site, with the minimum of the free energy located close to the inactive state distance between TM3 and TM6. The TM3-TM6 distance could be as large as $1.5 \mathrm{~nm}$ when the ligand binding site was in the active conformation, an observation compatible with basal activity. Agonist binding led to the stabilization of the TM5 bulge in the orthosteric site (Figure 4b). Although both inactive and active conformations remained accessible in the presence of the ligand, the minimum of the TM3-TM6 distance was shifted toward a more active-like state. A remarkable longrange allosteric coupling $(>2 \mathrm{~nm})$ between the orthosteric and $\mathrm{G}$ protein binding sites was hence captured by our simulations. The $0.5 \mathrm{~nm}$ outward movement of TM6 at the minimum of the landscape (Figure 4a,b) is smaller than that observed in active crystal structures, in agreement with experiments demonstrating that the fully active conformation can be stabilized only in the presence of an intracellular partner. ${ }^{51,23}$

Propagation of Activation through Microswitches. Structural changes in the orthosteric site of the $\beta_{2} \mathrm{AR}$ (Figure $4 \mathrm{~s}$ ) have been proposed to propagate toward the intracellular part via a "connector" centered around Ile $121^{3.40}$ and Phe $282^{6.44}$ (Figure 4t). ${ }^{6}$ Whereas we found the TM5 bulge and outward movement of TM6 to be loosely coupled, the free energy landscapes demonstrated that changes in the orthosteric site have a direct influence on the connector region (Figure $4 \mathrm{~d}, \mathrm{e})$. In the absence of an agonist, both inactive and active conformations of the connector were populated and the connector region could assume an active-like state even if the TM5 bulge was inactive (Figure 4d). In contrast, agonist binding resulted in a single free energy minimum where both the TM5 bulge and the connector were constrained to their active conformations (Figure $4 \mathrm{e}$ ).

From the connector region, activation is propagated via several conserved motifs (Figure $4 \mathrm{~g}, \mathrm{~h}, \mathrm{j}, \mathrm{k}, \mathrm{m}, \mathrm{n}, \mathrm{u}$ ). ${ }^{3}$ To investigate the communication between microswitches in the core of the TM region, we analyzed if the connector was coupled to solvation of the cavity surrounding Asp $79^{2.50}$, to the conformation of the NPxxY motif, and to the conformation of the $\mathrm{Y}-\mathrm{Y}$ motif. In the apo state, an inactive connector region was tightly coupled to a hydrated cavity with approximately $10-17$ waters (Figure $4 \mathrm{~g}$ ) and an inactive 


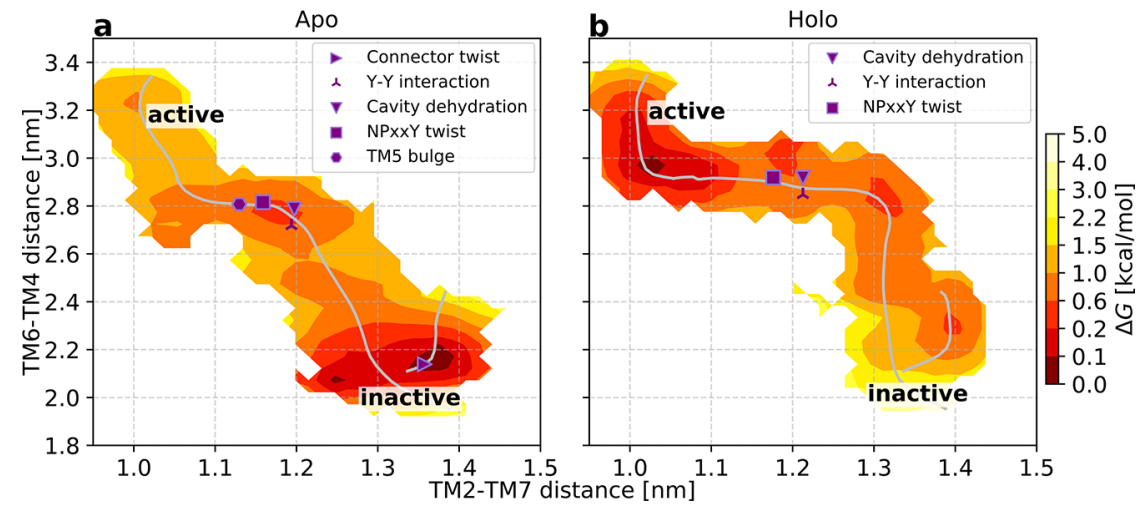

Figure 3. Free energy landscapes are projected onto the first two CVs used to optimize the minimum free energy path. The minimum free energy pathways were optimized (a) in the absence and (b) in the presence of the bound agonist. Characteristic events occurring during activation (see Table S3 for their definition) are marked on the string. The active and inactive labels mark the regions close to the active and inactive structures (PDB entries $3 \mathrm{POG}^{6}$ and $2 \mathrm{RH} 1,5$ respectively).

NPxxY motif (Figure 4j). This result is consistent with a highresolution crystal structure of an inactive $\beta_{1}$ adrenergic receptor (PDB entry $4 \mathrm{BVN}$ ), in which an ordered solvent network in this region was identified. ${ }^{17}$ In contrast, the active conformation of the connector was compatible with both the fully hydrated cavity and a desolvated state with only four or five water molecules as well as an inactive and active NPxxY motif. Interestingly, the connector configuration was more tightly coupled to the $\mathrm{Y}-\mathrm{Y}$ motif. An inactive connector was coupled to a broken $\mathrm{Y}-\mathrm{Y}$ interaction, whereas it was in the intermediate state or fully formed with an active connector (Figure $4 \mathrm{~m}$ ).

The free energy landscapes suggested that binding of an agonist reduces the number of inactive-like states of the Asp79 2.50 cavity, NPxxY, and Y-Y motifs (Figure 4h,k,n) as well as the orientation of Met82 2.53 (Figure S11), a residue located one helix turn above Asp $79^{2.50}$ that has been the focus of nuclear magnetic resonance (NMR) studies. ${ }^{52}$ For the Asp79.50 cavity and NPxxY motif, both active and inactive conformations were accessible in the presence of an agonist, but the active conformations were more favored energetically (Figure S13). The presence of an agonist resulted in an energy landscape with one active-like and two intermediate distances of the $\mathrm{Y}-\mathrm{Y}$ motif. The two latter minima were shifted by approximately $0.1 \mathrm{~nm}$ toward the active conformation compared to the apo state (Figure $4 n$ ).

The final combination of microswitches connected the $\mathrm{Y}-\mathrm{Y}$ motif to the motion of TM6 (Figure 4p,q). In the apo state, there was a relatively tight coupling between the $\mathrm{Y}-\mathrm{Y}$ interaction and the TM3-TM6 distance, with several metastable states lining the minimum free energy pathway between inactive and active conformations (Figure $4 p$ ). Agonist binding did not alter the coupling between these two microswitches but generally tilted the free energy landscape toward more active states along both of these dimensions (Figure 4q).

Access to the free energy landscapes in the absence and presence of a ligand thus enabled us to characterize the involvement of each microswitch in the transmission of allosteric coupling between the orthosteric ligand and the intracellular partner binding sites.

State-Dependent Sodium Binding. Several structures of class A GPCRs determined in the inactive state have revealed a sodium ion bound to the conserved Asp79 ${ }^{2.50}$ (Figure 5a). ${ }^{18}$
Binding of sodium to this residue has also been studied for several class A GPCRs with simulations. ${ }^{53-55}$ To investigate potential interactions between Asp7 $9^{2.50}$ and sodium ions, which were initially randomly added at physiological concentrations to the simulation system, we calculated the free energy landscape as a function of TM6 displacement and the distance between Asp79.50 and the closest sodium ion (Figure $5 b, c$ and Figure $S 11 j-1$ ). In the apo form, five metastable states were identified (Figure $5 b$ ). In the active-like conformation of TM6, the closest sodium interacted with a specific site in the second extracellular loop. Notably, sodium ions have been confirmed to bind in this pocket in crystal structures of adrenergic receptors (Figure 5a). ${ }^{11}$ In an intermediate conformation of TM6, the closest sodium was either bound to the second extracellular loop or descended into the binding site and formed a salt bridge to Asp $113^{3.32}$. Finally, in the completely inactive state of TM6, the closest sodium ion remained bound to Asp $113^{3.32}$ or was located in the Asp $79^{2.50}$ cavity. Sodium was hence only present in the Asp79 ${ }^{2.50}$ cavity when TM6 had completely relaxed to an inactive conformation, and even small increases of the TM3TM6 distance were incompatible with ion binding to this site. In the agonist-bound receptor with Asp $113^{3.32}$ ionized, sodium remained strongly bound to the second extracellular loop irrespective of the TM3-TM6 distance (Figure 5c). This difference is likely due to access to the binding site via the extracellular side being blocked by the bound ligand ${ }^{19,53,55}$ but could also be a result of conformational changes induced by the agonist. Spontaneous $\mathrm{Na}^{+}$binding to appropriate protein regions further confirms the relevance of the conformational sampling enabled by our computational protocol.

Impact of Asp79 $9^{2.50}$ Protonation. Protonation of two ionizable residues, Asp $79^{2.50}$ and Asp $130^{3.49}$, has been proposed to be involved in GPCR activation. ${ }^{20,31,56}$ In particular, MD simulations have indicated that Asp $79^{2.50}$, the most conserved residue among class A GPCRs, has a $\mathrm{p} K_{\mathrm{a}}$ value close to physiological $\mathrm{pH}$ and that the ionization state of this residue changes upon activation. ${ }^{19,20}$ As a previous simulation study of the $\beta_{2}$ AR showed that Asp79 2.50 (but not Asp130 ${ }^{3.49}$ ) may alter the activation pathway, ${ }^{31}$ we repeated the calculations of the minimum free energy pathway of activation with this residue in its protonated (neutral) form (Figure S7).

The free energy landscapes describing changes in the orthosteric site were similar to those obtained in simulations 

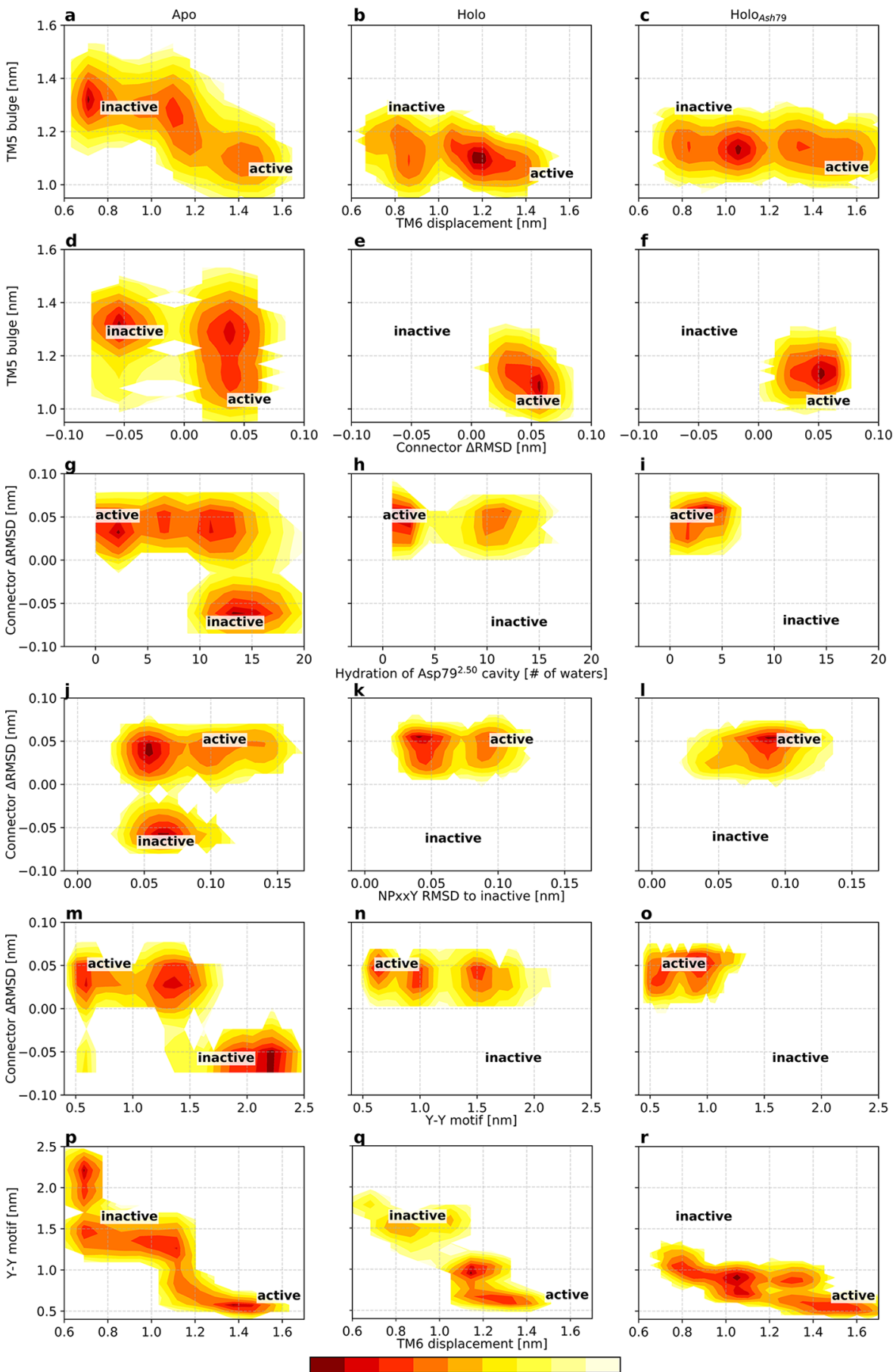

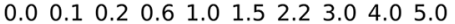

$\Delta G[\mathrm{kcal} / \mathrm{mol}]$
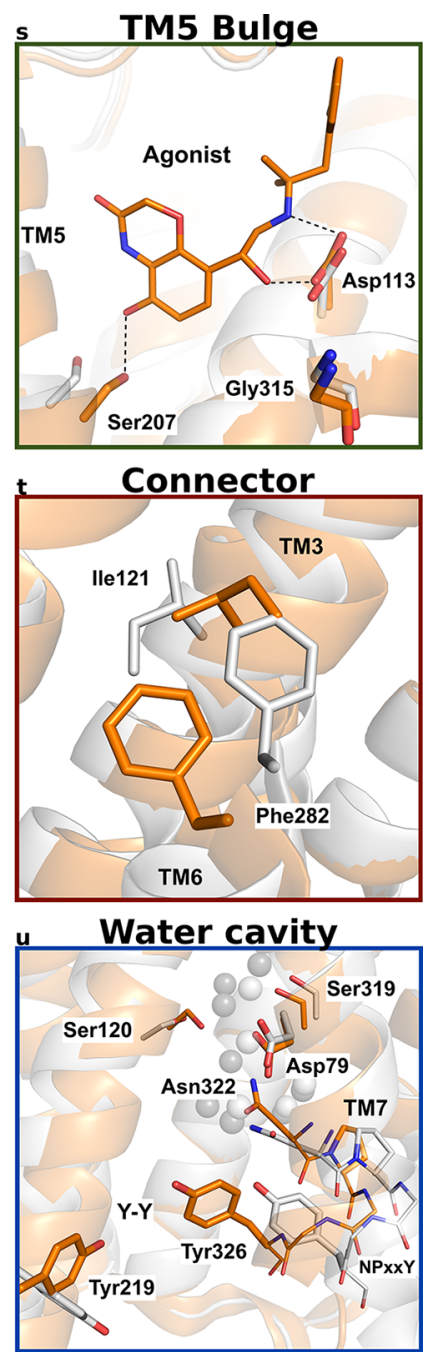

v TM6 displacement

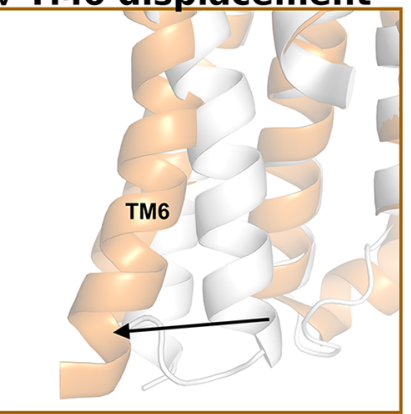

Figure 4. Free energy landscapes projected along variables of interest highlight changes in the pairwise coupling of microswitches following binding of an agonist ligand (middle column) and protonation of conserved residue Asp79.50 (right column). The free energy landscapes are projected

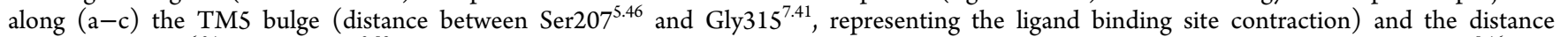
between Leu272 $2^{6.34}$ and $\operatorname{Arg} 131^{3.50}$, representing the outward movement of TM6; $(\mathrm{d}-\mathrm{f}$ ) the TM5 bulge (distance between Ser207.46 and Gly $315^{7.41}$ ) and the difference between the RMSD of Ile $121^{3.40}$ and Phe282 2.44 heavy atoms to the active and inactive crystal structures, ${ }^{5,6}$ representing the connector region $\triangle \mathrm{RMSD} ;(\mathrm{g}-\mathrm{i})$ the connector region $\triangle \mathrm{RMSD}$ and the number of water molecules within $0.8 \mathrm{~nm}$ of Asp792.50, representing the hydration of the Asp79 ${ }^{2.50}$ cavity; $(j-1)$ the connector region $\Delta$ RMSD and the RMSD of the NPxxY motif relative to the inactive structure $2 \mathrm{RH} 1 ;(\mathrm{m}-\mathrm{o})$ the connector region $\triangle \mathrm{RMSD}$ and the distance between the two tyrosines implicated in the $\mathrm{Y}-\mathrm{Y}$ interaction $(\mathrm{Y}-\mathrm{Y}$ motif), and $(\mathrm{p}-\mathrm{r})$ the $\mathrm{Y}-\mathrm{Y}$ motif distance and the displacement of TM6. Active and inactive state regions are labeled for each variable pair. Low-free energy regions are colored red, and high-free energy regions light yellow. Free energies are reported in kilocalories per mole. See Table S2 for microswitch definitions. ( $\mathrm{s}-\mathrm{v})$ Vignettes showing the conformation of the different microswitches in the active and inactive structures 3P0G

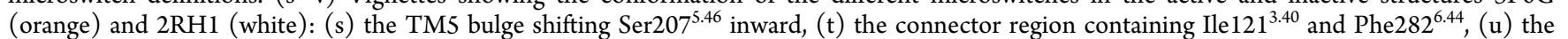
Asp79 $9^{2.50}$ cavity, NPxxY motif, and the two tyrosines, Tyr219 1.58 and Tyr326 $6^{7.53}$, of the Y-Y motif, and (v) the outward movement of TM6 upon activation. 


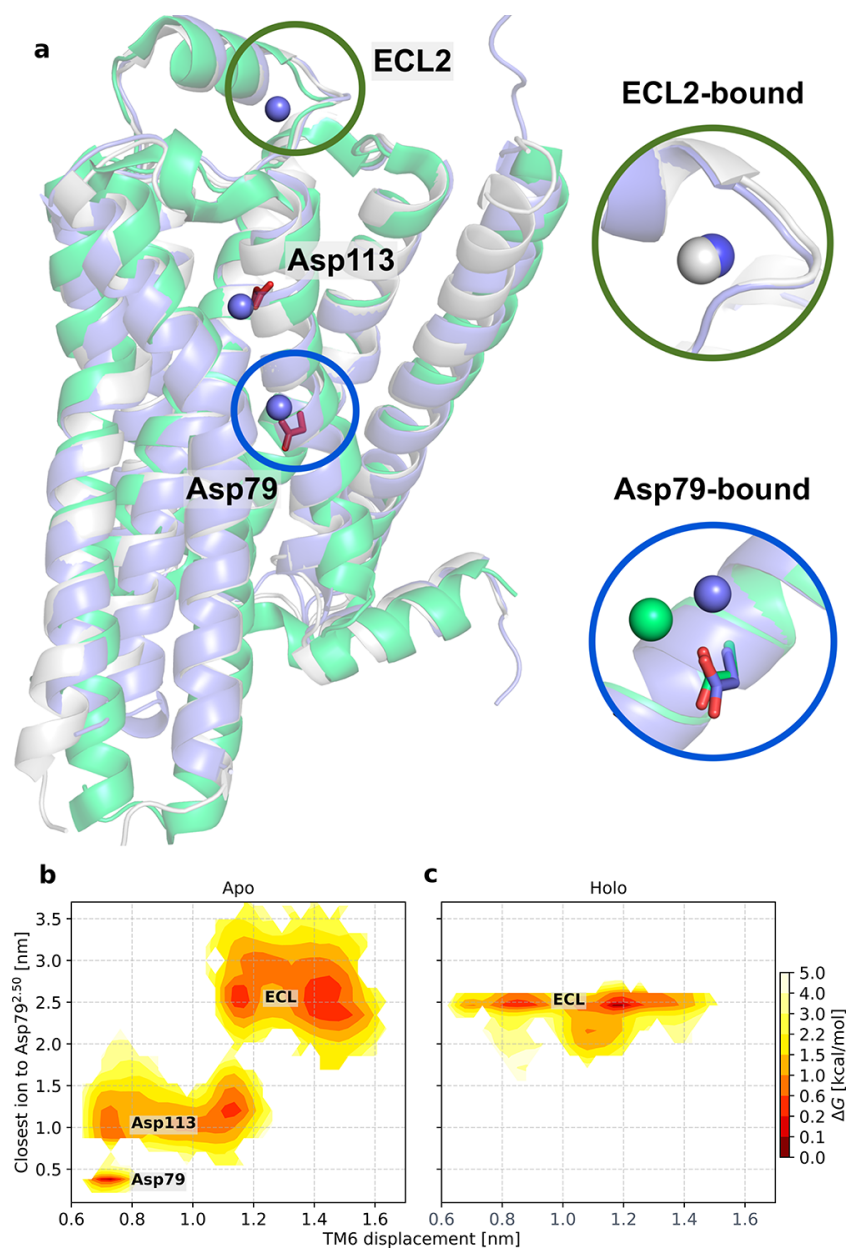

Figure 5. Sodium ions bind to three sites in a state-dependent manner. (a) The inactive $\beta_{1} \mathrm{AR}$ (PDB entry $4 \mathrm{BVN}^{17}$ ) and $\beta_{2} \mathrm{AR}(\mathrm{PDB}$ entry $\left.4 \mathrm{LDE}^{11}\right)$ structures and a representative inactive state simulation snapshot are shown as green, white, and blue cartoons, respectively. Representative positions of sodium ions in the MD simulations are shown as blue spheres. In the insets, a comparison to the positions of sodium ions found in the crystal structures of active $\beta_{2} \mathrm{AR}$ (white sphere) and inactive $\beta_{1} \mathrm{AR}$ (white spheres) is shown. (b and c) Free energy landscapes along the TM6 displacement and the shortest distance between Asp79 $79^{2.50}$ and a sodium ion for the apo and holo simulations.

with Asp79 2.50 ionized (Figure 4c,f). There was a weak coupling between the TM5 bulge and the intracellular region, with two major energy wells describing the conformation of TM6. Compared to the agonist-bound receptor with Asp79 2.50 ionized, the minima were shifted further toward active-like conformations for the protonated state (Figure 4c). The TM5 bulge remained strongly coupled to conformational changes observed in the connector region irrespective of the ionization state of Asp79 2.50 (Figure 4e,f). The strongest effects of Asp $79^{2.50}$ protonation were observed for the hydrated cavity surrounding this residue (Figure 4h,i), NPxxY (Figure 4k,l), and $\mathrm{Y}-\mathrm{Y}$ motifs (Figure $4 \mathrm{n}, \mathrm{o}$ ). Whereas the free energy landscapes showed that both active- and inactive-like conformations of these switches were populated in simulations with ionized Asp $79^{2.50}$, the protonated state resulted only in energy wells close to the active conformation (Figure 4i,l,o). It was also evident that TM6 was stabilized in more active-like conformations by the protonated Asp79 ${ }^{2.50}$ (Figure 4r). Interestingly, intermediate states in which the $\mathrm{Y}-\mathrm{Y}$ motif, as well as the NPxxY, adopted an active-like conformation and a small TM6 displacement were also populated (Figure 4r and Figure S13). Such an intermediate state has been observed, albeit rarely, in simulations considering a protonated Asp $79^{2.50} 31$

Comparison of representative structures from the simulations of ionized and protonated Asp $79^{2.50}$ in active-like states revealed that two distinct conformations of the NPxxY motif were obtained (Figure 6). The simulations carried out with

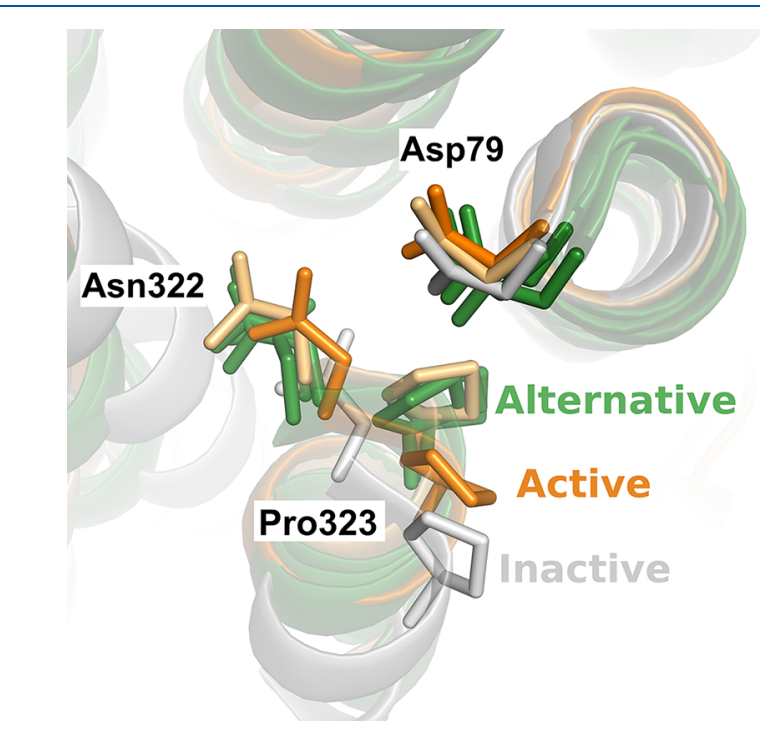

Figure 6. Alternative conformation of the NPxxY identified along the most probable pathway calculated with a protonated Asp $79^{2.50}$. A representative simulation snapshot of active-like states of the $\beta_{2} \mathrm{AR}$ with Asp $79^{2.50}$ ionized and protonated are colored orange and light orange, respectively. A representative simulation snapshot of the inactive-like state of the $\beta_{2} \mathrm{AR}$ is colored white. The structural comparison highlights the resemblance between an alternative conformation of the $\mathrm{NPxxY}$ motif (light orange) that is favored by Asp $79^{2.50}$ protonation in the simulations and observed in three crystal structures of other class A GPCRs. Crystal structures of the other GPCRs are colored green (PDB entries 6DRX, ${ }^{57}$ agonist-bound 5$\mathrm{HT}_{2 B}$ serotonin receptor; $3 \mathrm{QAK}, 59$ agonist-bound $\mathrm{A}_{2 A}$ adenosine receptor; and $6 \mathrm{DO} 1,{ }^{60}$ angiotensin II type 1 receptor in an active conformation).

ionized Asp79 $9^{2.50}$ favored structures that were more similar to the crystal structure of the active $\beta_{2} \mathrm{AR}$. An alternative conformation of the active NPxxY motif appeared for the protonated Asp $79^{2.50}$, which was not favored energetically in the simulations of the ionized form (Figures S10k,l and S13). Although this conformation of the NPxxY motif did not match any $\beta_{2} \mathrm{AR}$ crystal structure, it was strikingly similar to conformations observed in crystal structures of other class $A$ GPCRs in either agonist-bound (serotonin 5- $\mathrm{HT}_{2 B}$ and $\mathrm{A}_{2 A}$ adenosine receptors) or active (angiotensin II type 1 ) conformations (Figure 6). ${ }^{57,58}$ Our protocol thus allowed us to sample metastable states not yet resolved by experimental structure determination that may play a role in activation.

Identified CVs Capture Activation of Many Class A GPCRs. Efficient characterization of free energy landscapes with the string method relies on selection of appropriate CVs, which is a nontrivial task. Here, CVs were derived from a conventional $\mathrm{MD}$ trajectory of $\beta_{2} \mathrm{AR}$ deactivation in a datadriven fashion. Considering that the conformational changes involved in class A GPCR activation are largely conserved, ${ }^{3}$ we 
explored the possibility of transferring the CVs to the conformational sampling of other GPCRs. We mapped the CVs identified for $\beta_{2} \mathrm{AR}$ to 10 other class A GPCRs with active and inactive structures available (Figure 7). Strikingly, the
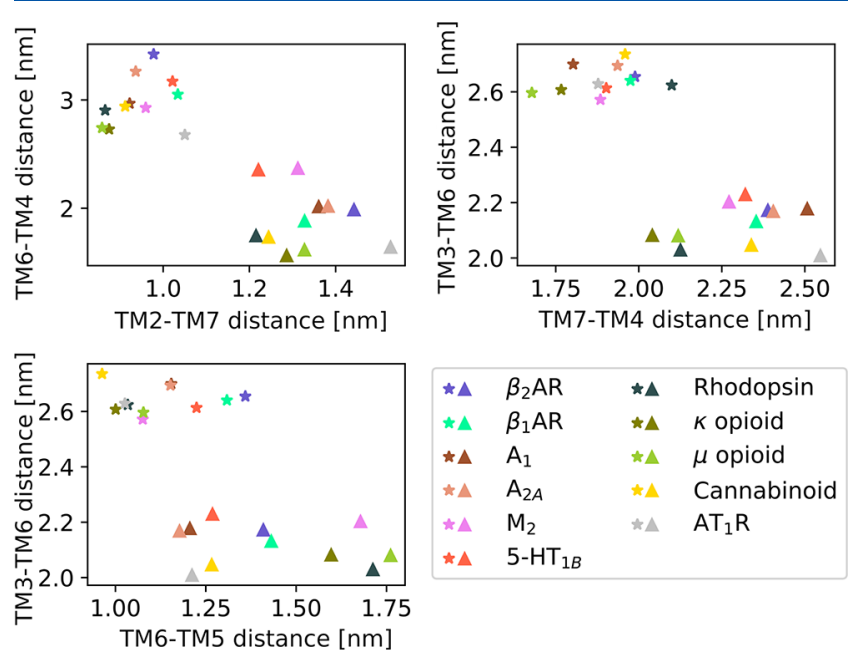

$\begin{array}{llll}\star \Delta & \beta_{2} \mathrm{AR} & \star \Delta & \text { Rhodopsin } \\ \star \Delta & \beta_{1} \mathrm{AR} & \star \Delta & \kappa \text { opioid } \\ \star \Delta & \mathrm{A}_{1} & \star \Delta & \mu \text { opioid } \\ \star \Delta & \mathrm{A}_{2 A} & \star \Delta & \text { Cannabinoid } \\ \star \Delta & \mathrm{M}_{2} & \star \Delta & \mathrm{AT}_{1} \mathrm{R} \\ \star \Delta & 5-\mathrm{HT}_{1 B} & & \\ & & & \\ & & & \end{array}$

Figure 7. Active and inactive class A GPCR structures cluster into two distinct groups in the five-dimensional $\mathrm{CV}$ space used in this work. $\beta_{2} \mathrm{AR}$ and 10 active (stars) and inactive (triangles) structures of other class A GPCRs are projected onto the five original CVs. Details of the mapping can be found in Table S4. Their clustering into two groups highlights that the activation mechanism of all class A GPCRs can likely be described by the CVs identified in this work.

active and inactive structures clearly separate into two distinct clusters. Together with the fact that we could capture relevant receptor conformations that were not observed in the MD trajectory used to identify CVs (Figure 6), this indicates that our approach could potentially be used to characterize the activation of many class A GPCRs.

\section{DISCUSSION}

Experimental structures of the $\beta_{2} \mathrm{AR}$ in inactive and active conformations provide a basis for the molecular understanding of GPCR signaling. However, it has become increasingly clear that the static structures do not capture all functionally relevant states involved in activation of these molecular machines. ${ }^{26,27,32,33}$ In a pioneering study, Dror et al. gained insights into the activation pathway of the $\beta_{2} \mathrm{AR}$ from long time scale $\mathrm{MD}$ simulations. ${ }^{31}$ Despite this computational tour de force enabled by the development of hardware specialized for $\mathrm{MD}$, these simulations did not allow quantification of the accessibility of different conformational states. The approach proposed in this work builds on the data generated by Dror et al. and on methodologies suggested in previous molecular simulation studies ${ }^{27,29,30,33}$ to further allow the assessment of the impact of agonist binding on microswitches involved in activation.

Our work recapitulates a number of key findings of previous enhanced sampling ${ }^{27,29,30,32,33}$ and long time scale simulations of GPCRs. ${ }^{31}$ In agreement with previous work, our simulations revealed a complex conformational landscape of the $\beta_{2} \mathrm{AR}$ with only weak coupling between the othosteric site and G protein binding site. Through the analysis of the free energy landscapes, we could quantify the coupling between spatially connected microswitches in the transmembrane region, which ranged from strong to relatively weak and was influenced by protonation of Asp $79^{2.50}$. Our study also further illustrates that the energy landscapes depend on the variables chosen to project the conformational states. This is not an artifact of the protocol but rather an inherent limitation of dimensionality reduction. In particular, it is clear that considering only three states along the activation path (active, intermediate, and inactive states) does not allow us to capture the complexity of the conformational changes induced by ligand binding. $^{26,27,32,33}$ This work, through the resolution of the complete conformational ensemble lining the most probable activation pathway, can aid in determining the placement of spectroscopic probes to monitor the distribution of specific microswitches under different conditions.

Our work shows agreement with spectroscopy studies, although a quantitative comparison remains challenging. Whereas the $\beta_{2} \mathrm{AR}$ crystal structure solved in complex with an agonist in the absence of an intracellular partner (e.g., G protein or G protein-mimicking nanobody) is similar to those determined with antagonists, ${ }^{36}$ NMR spectroscopy experiments have demonstrated that agonist binding stabilizes other conformations in certain parts of the TM region, e.g., close to Met215 5.54 and Met82 2.53.52 For example, the region surrounding Met $82^{2.53}$, located one helical turn above Asp $79^{2.50}$, was shown to adopt three conformations in the prescence of a neutral antagonist (with signaling properties similar to those of the apo receptor). A bound agonist, on the other hand, restricted this region to a single active-like state. $^{51,52}$ Similar to these experiments, our free energy landscapes demonstrate that several conformations of the connector, Asp $79^{2.50}$ cavity, and the orientation of Met $82^{2.53}$ are available under the apo conditions (Figures $\mathrm{S} 10 \mathrm{~g}-\mathrm{i}, \mathrm{m}-\mathrm{o}$ and $S 11 d-f)$. In particular, Figure $S 11 d-f$ shows three conformational Met82 2.53 states of comparable free energy for the apo receptor, whereas the landscape is significantly shifted to favor a single active state conformation for the agonist-bound receptor. Protonation of Asp $79^{2.50}$ further increases this shift. In the presence of an agonist, the connector is locked in a single state and a desolvated state of the Asp $79^{2.50}$ cavity is stabilized, which creates a more activelike receptor conformation in the vicinity of Met82 2.53 . In agreement with the NMR data, we also find that the agonist cannot stabilize the fully active conformation of the receptor and that TM6 accesses several intermediate conformations that are distinct from those observed in crystal structures.

The rapidly increasing number of crystal structures provides insights into how agonist binding to GPCRs, despite recognizing disparate molecules, can result in very similar conformational changes. The bulge of TM5, which we found to be strongly coupled to the connector region, is also present in active state structures of other GPCRs. ${ }^{61,62}$ Whereas this conformational change is the result of a direct interaction with TM5 in the case of the $\beta_{2} \mathrm{AR}$, interactions of the agonist with other helices appear to indirectly influence the same region in the case of the $\mu$ opioid and $A_{2 A}$ adenosine receptor. ${ }^{81,62}$ In other cases, the connector region was found to have a similar arrangement in the active and inactive states (e.g., the $\mathrm{M}_{2}$ muscarinic receptor) ${ }^{63}$ In such cases, receptor activation may be controlled by direct modulation of the Asp $79^{2.50}$ cavity, e.g., via the conserved Trp286 6.48 . Several recent experimental studies have demonstrated that Asp $79^{2.50}$ and residues forming the hydrated TM cavity play an important role in signaling and can even steer activation via $G$ protein-dependent and $G$ protein-independent pathways. ${ }^{25,64,65}$ One mechanism by 
which Asp $79^{2.50}$ could control the receptor conformation is via its protonation state. Agonist binding destabilizes the water network in the solvated TM cavity, which may lead to a larger population of protonated Asp $79^{2.50}$ and disrupt binding of sodium to this pocket. ${ }^{19,20}$ In turn, the protonated Asp79 2.50 stabilizes a structure of the NPxxY that has been observed for other class A GPCRs crystallized in active and active-like states, suggesting that this alternative conformation of TM7 may be relevant for function. NMR experiments have shown that different ligands stabilize different active states. ${ }^{66}$ In particular, agonists that preferentially signal via arrestin mainly affect the conformation of TM7, whereas a neutral agonist stabilizes both the $G$ protein and the arrestin signaling state. While two distinct active state conformations of the NPxxY motif were identified in our simulations for a neutral agonist, a ligand with different biased signaling properties may preferentially stabilize one of these states. Interestingly, the ionized and protonated forms of Asp $79^{2.50}$ also stabilize different TM6 conformations, which could change the intracellular interface that interacts with G proteins and arrestins. ${ }^{9}$ These results, combined with the fact that Asp79 2.50 is the most conserved residue in the class A receptor family, support the idea that this region is a central hub for controlling receptor activation.

With the protocol developed herein, we sampled enough transitions along the activation path to obtain free energy profiles of GPCR activation by accumulating a few microseconds of total simulation time. Compared to regular MD simulations, the optimized string of swarms method can thus provide reliable energetic insights using 1-2 orders magnitude less simulation time than plain $\mathrm{MD}$ simulations ${ }^{31,32}$ and comparable to other enhanced sampling methods such as GaMD. ${ }^{26}$ From a practical point of view, the short trajectories in the swarms of trajectories method are easy to run in parallel with minimal communication overhead even in a heterogeneous computational environment. An important consideration that has guided our choice of enhanced sampling methodology is that the method has the advantage of functioning well in high-dimensional space, i.e., with many CVs. This is because we only optimize the one-dimensional string instead of opting to sample the entire landscape spanned by the CVs. This means we can utilize a high-dimensional CV space, thus alleviating the need to reduce the dimensionality of the conformational landscape to one or two dimensions, as is done in most CV-based methods such as umbrella sampling or metadynamics. ${ }^{67}$

On the other hand, a well-known limitation of the string with swarms method is that it is guaranteed to converge to the most probable path closest only to the initial guess of the path, and not necessarily the globally most probable path. The naive assumption of a straight initial path is not guaranteed to converge to the latter. Here we have proposed to alleviate this shortcoming by exploiting previous knowledge of the activation pathway and deriving an initial guess of the pathway likely to be close to the globally most probable path. An initial pathway can also be transferred from a similar system (as revealed in Figure 7) or inferred from available experimental data. If multiple pathways are nevertheless expected, the protocol presented herein provides the tools necessary to compare them: the swarms from separate string simulations can be included in the same transition matrix and used to compute a single free energy landscape. It is also worth noting that the relative free energies of the end point conformations (evaluated by integration over the end point basins) do not depend on the transition pathway and should be estimated correctly. The protocol is also applicable to complex transitions involving many intermediate states: in such a case, one may launch multiple strings to explore different parts of the activation path, and let every substring converge separately, eventually combining the transitions derived from them to yield a single free energy landscape. Finally, we note that the string of swarms method can also be used as a complementary method to instantiate Markov state model (MSM) simulations. $^{68}$

\section{CONCLUSION}

Despite the major progress in structural biology for GPCRs, many aspects of receptor function remain poorly understood. Here, we derived the most probable activation path of $\beta_{2} \mathrm{AR}$ using an improved variant of the string method with swarms of trajectories. The associated free energy landscapes revealed that agonist binding is only loosely connected to outward movement of TM6, while the coupling between microswitch pairs in the transmembrane region, in particular the TM5 bulge and the connector conformation, was strongly coupled to ligand binding. We also investigated the influence of sodium binding to known sodium interaction sites and found that a sodium ion travels along a distinct pathway from the Asp $79^{2.50}$ cavity via the orthosteric site as the apo receptor is activated. We then studied the effect of Asp79 ${ }^{2.50}$ protonation on the agonist-bound receptor and found that a protonated Asp $79^{2.50}$ did not shift the free energy landscape to favor active-like conformations; it also led to a distinct conformational state of the NPxxY motif. This protonated active state was reminiscent of active structures of other class A GPCRs, suggesting that protonation of this conserved residue is a shared feature across several GPCRs. We expect that insights from atomistic MD simulations will continue to be valuable tools for interpreting experimental data. In particular, we anticipate that our methodology will enable further insights into how binding of different ligands influences the conformational landscape, potentially making it possible to design ligands with biased signaling properties. ${ }^{25}$ The method is equally well suited to study the effect of allosteric modulators and the influence of different lipidic environments. ${ }^{69}$ As the same approach can likely be transferred to other class A GPCRs, future applications will shed light on the common principles of activation as well as on the details that give each receptor a unique signaling profile, paving the way to the design of more effective drugs.

\section{ASSOCIATED CONTENT}

\section{SI Supporting Information}

The Supporting Information is available free of charge at https://pubs.acs.org/doi/10.1021/acs.biochem.9b00842.

Tables S1-S4, Supplementary Methods (system preparation, $\mathrm{MD}$ simulation details, $\mathrm{CV}$ selection, swarms of trajectories simulation setup, algorithmic improvements to the swarms of trajectories method, and free energy computation), and Figures S1-S13 (PDF)

\section{AUTHOR INFORMATION}

\section{Corresponding Author}

Lucie Delemotte - Science for Life Laboratory, Department of Applied Physics, KTH Royal Institute of Technology, SE-100 44 
Stockholm, Sweden; 이이이.org/0000-0002-0828-3899; Email: lucie.delemotte@scilifelab.se

\section{Authors}

Oliver Fleetwood - Science for Life Laboratory, Department of Applied Physics, KTH Royal Institute of Technology, SE-100 44 Stockholm, Sweden; 이이.org/0000-0002-4277-2661

Pierre Matricon - Science for Life Laboratory, Department of Cell and Molecular Biology, Uppsala University, SE-751 05 Uppsala, Sweden; 이이이.org/0000-0001-9350-896X

Jens Carlsson - Science for Life Laboratory, Department of Cell and Molecular Biology, Uppsala University, SE-751 05 Uppsala, Sweden; (1) orcid.org/0000-0003-4623-2977

Complete contact information is available at: https://pubs.acs.org/10.1021/acs.biochem.9b00842

\section{Funding}

This work was supported by grants from the Göran Gustafsson Foundation and Science for Life Laboratory to J.C. and L.D. This work was also supported by grants from the Swedish Research Council (2017-4676) and the Swedish strategic research program eSSENCE to J.C.

\section{Notes}

The authors declare no competing financial interest.

\section{ACKNOWLEDGMENTS}

The simulations were performed on resources provided by the Swedish National Infrastructure for Computing (SNIC) at the PDC Centre for High Performance Computing (PDC-HPC). The authors thank D. E. Shaw Research for providing access to their MD trajectories.

\section{ABBREVIATIONS}

$\mathrm{MD}$, molecular dynamics; GPCR, G protein-coupled receptor; $\mathrm{CV}$, collective variable; $\beta_{2} \mathrm{AR}, \beta_{2}$ adrenergic receptor; $\mathrm{PDB}$, Protein Data Bank.

\section{REFERENCES}

(1) Fredriksson, R., Lagerström, M. C., Lundin, L.-G., and Schiöth, H. B. (2003) The G-protein-coupled receptors in the human genome form five main families. Phylogenetic analysis, paralogon groups, and fingerprints. Mol. Pharmacol. 63, 1256-1272.

(2) Hauser, A. S., Attwood, M. M., Rask-Andersen, M., Schiöth, H. B., and Gloriam, D. E. (2017) Trends in GPCR drug discovery: new agents, targets and indications. Nat. Rev. Drug Discovery 16, 829-842.

(3) Weis, W. I., and Kobilka, B. K. (2018) The Molecular basis of G protein-coupled receptor activation. Annu. Rev. Biochem. 87, 897919.

(4) Manglik, A., and Kruse, A. C. (2017) Structural Basis for G Protein-Coupled Receptor Activation. Biochemistry 56, 5628-5634.

(5) Cherezov, V., Rosenbaum, D. M., Hanson, M. A., Rasmussen, S. G. F., Thian, F. S., Kobilka, T. S., Choi, H.-J., Kuhn, P., Weis, W. I., Kobilka, B. K., and Stevens, R. C. (2007) High-Resolution Crystal Structure of an Engineered Human 2-Adrenergic G Protein-Coupled Receptor. Science 318, 1258-1265.

(6) Rasmussen, S. G., Choi, H.-J., Fung, J. J., Pardon, E., Casarosa, P., Chae, P. S., DeVree, B. T., Rosenbaum, D. M., Thian, F. S., Kobilka, T. S., et al. (2011) Structure of a nanobody-stabilized active state of the $\beta 2$ adrenoceptor. Nature 469, 175-180.

(7) Rasmussen, S. G., DeVree, B. T., Zou, Y., Kruse, A. C., Chung, K. Y., Kobilka, T. S., Thian, F. S., Chae, P. S., Pardon, E., Calinski, D., Mathiesen, J. M., Shah, S. T. A., Lyons, J. A., Caffrey, M., Gellman, S. H., Steyaert, J., Skiniotis, G., Weis, W. I., Sunahara, R. K., and Kobilka, B. K. (2011) Crystal structure of the $\beta 2$ adrenergic receptor-Gs protein complex. Nature 477, 549-555.
(8) Masureel, M., Zou, Y., Picard, L.-P., van der Westhuizen, E., Mahoney, J. P., Rodrigues, J. P. G. L. M., Mildorf, T. J., Dror, R. O., Shaw, D. E., Bouvier, M., Pardon, E., Steyaert, J., Sunahara, R. K., Weis, W. I., Zhang, C., and Kobilka, B. K. (2018) Structural insights into binding specificity, efficacy and bias of a $\beta 2 \mathrm{AR}$ partial agonist. Nat. Chem. Biol. 14, 1059-1066.

(9) Staus, D. P., Strachan, R. T., Manglik, A., Pani, B., Kahsai, A. W., Kim, T. H., Wingler, L. M., Ahn, S., Chatterjee, A., Masoudi, A., Kruse, A. C., Pardon, E., Steyaert, J., Weis, W. I., Prosser, R. S., Kobilka, B. K., Costa, T., and Lefkowitz, R. J. (2016) Allosteric nanobodies reveal the dynamic range and diverse mechanisms of Gprotein-coupled receptor activation. Nature 535, 448-452.

(10) Liu, X., Ahn, S., Kahsai, A. W., Meng, K.-C., Latorraca, N. R., Pani, B., Venkatakrishnan, A. J., Masoudi, A., Weis, W. I., Dror, R. O., Chen, X., Lefkowitz, R. J., and Kobilka, B. K. (2017) Mechanism of intracellular allosteric $\beta 2 \mathrm{AR}$ antagonist revealed by $\mathrm{X}$-ray crystal structure. Nature 548, 480-484.

(11) Ring, A. M., Manglik, A., Kruse, A. C., Enos, M. D., Weis, W. I., Garcia, K. C., and Kobilka, B. K. (2013) Adrenaline-activated structure of $\beta 2$-adrenoceptor stabilized by an engineered nanobody. Nature 502, 575-579.

(12) Hanson, M. A., Cherezov, V., Griffith, M. T., Roth, C. B., Jaakola, V.-P., Chien, E. Y., Velasquez, J., Kuhn, P., and Stevens, R. C. (2008) A Specific Cholesterol Binding Site Is Established by the $2.8 \AA$ Structure of the Human $\beta 2$-Adrenergic Receptor. Structure 16, 897905.

(13) Wacker, D., Fenalti, G., Brown, M. A., Katritch, V., Abagyan, R., Cherezov, V., and Stevens, R. C. (2010) Conserved Binding Mode of Human $\beta 2$ Adrenergic Receptor Inverse Agonists and Antagonist Revealed by X-ray Crystallography. J. Am. Chem. Soc. 132, 1144311445 .

(14) Rasmussen, S. G. F., DeVree, B. T., Zou, Y., Kruse, A. C., Chung, K. Y., Kobilka, T. S., Thian, F. S., Chae, P. S., Pardon, E., Calinski, D., Mathiesen, J. M., Shah, S. T. A., Lyons, J. A., Caffrey, M., Gellman, S. H., Steyaert, J., Skiniotis, G., Weis, W. I., Sunahara, R. K., and Kobilka, B. K. (2007) Crystal structure of the human $\beta 2$ adrenergic G-protein-coupled receptor. Nature 450, 383-387.

(15) García-Nafría, J., and Tate, C. G. (2019) Cryo-EM structures of GPCRs coupled to Gs, Gi and Go. Mol. Cell. Endocrinol. 488, 1-13.

(16) Ballesteros, J. A., and Weinstein, H. (1995) Methods in Neurosciences, pp 366-428, Elsevier.

(17) Miller-Gallacher, J. L., Nehme, R., Warne, T., Edwards, P. C., Schertler, G. F., Leslie, A. G., and Tate, C. G. (2014) The $2.1 \AA$ resolution structure of cyanopindolol-bound $\beta 1$-adrenoceptor identifies an intramembrane $\mathrm{Na}+$ ion that stabilises the ligand-free receptor. PLoS One 9, e92727.

(18) Katritch, V., Fenalti, G., Abola, E. E., Roth, B. L., Cherezov, V., and Stevens, R. C. (2014) Allosteric sodium in class A GPCR signaling. Trends Biochem. Sci. 39, 233-244.

(19) Vickery, O. N., Carvalheda, C. A., Zaidi, S. A., Pisliakov, A. V., Katritch, V., and Zachariae, U. (2018) Intracellular Transfer of Na+ in an Active-State G-Protein-Coupled Receptor. Structure 26, 171180.e2.

(20) Ranganathan, A., Dror, R. O., and Carlsson, J. (2014) Insights into the Role of Asp79 2.50 in $\beta 2$ Adrenergic Receptor Activation from Molecular Dynamics Simulations. Biochemistry 53, 7283-7296.

(21) Latorraca, N. R., Venkatakrishnan, A., and Dror, R. O. (2017) GPCR dynamics: structures in motion. Chem. Rev. 117, 139-155.

(22) Lamichhane, R., Liu, J. J., Pljevaljcic, G., White, K. L., van der Schans, E., Katritch, V., Stevens, R. C., Wüthrich, K., and Millar, D. P. (2015) Single-molecule view of basal activity and activation mechanisms of the G protein-coupled receptor $\beta 2 \mathrm{AR}$. Proc. Natl. Acad. Sci. U. S. A. 112, 14254-14259.

(23) Manglik, A., Kim, T. H., Masureel, M., Altenbach, C., Yang, Z., Hilger, D., Lerch, M. T., Kobilka, T. S., Thian, F. S., Hubbell, W. L., Prosser, R. S., and Kobilka, B. K. (2015) Structural Insights into the Dynamic Process of $\beta 2$-Adrenergic Receptor Signaling. Cell 161, $1101-1111$. 
(24) Gregorio, G. G., Masureel, M., Hilger, D., Terry, D. S., Juette, M., Zhao, H., Zhou, Z., Perez-Aguilar, J. M., Hauge, M., Mathiasen, S., et al. (2017) Single-molecule analysis of ligand efficacy in $\beta 2 A R-G-$ protein activation. Nature 547, 68-73.

(25) Picard, L.-P., Schonegge, A.-M., and Bouvier, M. (2019) Structural Insight into G Protein-Coupled Receptor Signaling Efficacy and Bias between Gs and $\beta$-Arrestin. ACS Pharmacol Transl Sci 2, $148-154$.

(26) Miao, Y., and McCammon, J. A. (2016) Graded activation and free energy landscapes of a muscarinic G-protein-coupled receptor. Proc. Natl. Acad. Sci. U. S. A. 113, 12162-12167.

(27) Bhattacharya, S., and Vaidehi, N. (2010) Computational Mapping of the Conformational Transitions in Agonist Selective Pathways of a G-Protein Coupled Receptor. J. Am. Chem. Soc. 132, 5205-5214.

(28) Niesen, M. J. M., Bhattacharya, S., and Vaidehi, N. (2011) The Role of Conformational Ensembles in Ligand Recognition in GProtein Coupled Receptors. J. Am. Chem. Soc. 133, 13197-13204.

(29) Shan, J., Khelashvili, G., Mondal, S., Mehler, E. L., and Weinstein, H. (2012) Ligand-Dependent Conformations and Dynamics of the Serotonin 5-HT2A Receptor Determine Its Activation and Membrane-Driven Oligomerization Properties. PLoS Comput. Biol. 8, e1002473.

(30) Li, J., Jonsson, A. L., Beuming, T., Shelley, J. C., and Voth, G. A. (2013) Ligand-Dependent Activation and Deactivation of the Human Adenosine A2A Receptor. J. Am. Chem. Soc. 135, 8749-8759.

(31) Dror, R. O., Arlow, D. H., Maragakis, P., Mildorf, T. J., Pan, A. C., Xu, H., Borhani, D. W., and Shaw, D. E. (2011) Activation mechanism of the $\beta 2$-adrenergic receptor. Proc. Natl. Acad. Sci. U. S. A. 108, 18684-18689.

(32) Kohlhoff, K. J., Shukla, D., Lawrenz, M., Bowman, G. R., Konerding, D. E., Belov, D., Altman, R. B., and Pande, V. S. (2014) Cloud-based simulations on Google Exacycle reveal ligand modulation of GPCR activation pathways. Nat. Chem. 6, 15-21.

(33) Provasi, D., Artacho, M. C., Negri, A., Mobarec, J. C., and Filizola, M. (2011) Ligand-induced modulation of the free-energy landscape of $\mathrm{G}$ protein-coupled receptors explored by adaptive biasing techniques. PLoS Comput. Biol. 7, e1002193.

(34) Tikhonova, I. G., Selvam, B., Ivetac, A., Wereszczynski, J., and McCammon, J. A. (2013) Simulations of biased agonists in the $\beta 2$ adrenergic receptor with accelerated molecular dynamics. Biochemistry 52, 5593-5603.

(35) Harpole, T. J., and Delemotte, L. (2018) Conformational landscapes of membrane proteins delineated by enhanced sampling molecular dynamics simulations. Biochim. Biophys. Acta, Biomembr. 1860, 909-926.

(36) Rosenbaum, D. M., Zhang, C., Lyons, J. A., Holl, R., Aragao, D., Arlow, D. H., Rasmussen, S. G. F., Choi, H.-J., DeVree, B. T., Sunahara, R. K., Chae, P. S., Gellman, S. H., Dror, R. O., Shaw, D. E., Weis, W. I., Caffrey, M., Gmeiner, P., and Kobilka, B. K. (2011) Structure and function of an irreversible agonist- $\beta 2$ adrenoceptor complex. Nature 469, 236-240.

(37) Huang, J., Rauscher, S., Nawrocki, G., Ran, T., Feig, M., de Groot, B. L., Grubmüller, H., and MacKerell, A. D., Jr (2017) CHARMM36m: an improved force field for folded and intrinsically disordered proteins. Nat. Methods 14, 71-73.

(38) Jorgensen, W. L., Chandrasekhar, J., Madura, J. D., Impey, R. W., and Klein, M. L. (1983) Comparison of simple potential functions for simulating liquid water. J. Chem. Phys. 79, 926-935.

(39) Klauda, J. B., Venable, R. M., Freites, J. A., O'Connor, J. W., Tobias, D. J., Mondragon-Ramirez, C., Vorobyov, I., MacKerell, A. D., Jr, and Pastor, R. W. (2010) Update of the CHARMM all-atom additive force field for lipids: validation on six lipid types. J. Phys. Chem. B 114, 7830-7843.

(40) Lee, J., Cheng, X., Swails, J. M., Yeom, M. S., Eastman, P. K., Lemkul, J. A., Wei, S., Buckner, J., Jeong, J. C., Qi, Y., et al. (2016) CHARMM-GUI input generator for NAMD, GROMACS, AMBER, OpenMM, and CHARMM/OpenMM simulations using the
CHARMM36 additive force field. J. Chem. Theory Comput. 12, 405-413.

(41) Abraham, M. J., Murtola, T., Schulz, R., Páll, S., Smith, J. C., Hess, B., and Lindahl, E. (2015) GROMACS: High performance molecular simulations through multi-level parallelism from laptops to supercomputers. SoftwareX 1, 19-25.

(42) Tribello, G. A., Bonomi, M., Branduardi, D., Camilloni, C., and Bussi, G. (2014) PLUMED 2: New feathers for an old bird. Comput. Phys. Commun. 185, 604-613.

(43) Westerlund, A. M., and Delemotte, L. (2018) Effect of Ca2+ on the promiscuous target-protein binding of calmodulin. PLoS Comput. Biol. 14, e1006072.

(44) Pedregosa, F., Varoquaux, G., Gramfort, A., Michel, V., Thirion, B., Grisel, O., Blondel, M., Prettenhofer, P., Weiss, R., Dubourg, V., Vanderplas, J., Passos, A., Cournapeau, D., Brucher, M., Perrot, M., and Duchesnay, E. (2011) Scikit-learn: Machine Learning in Python. Journal of Machine Learning Research 12, 2825-2830.

(45) Montavon, G., Lapuschkin, S., Binder, A., Samek, W., and Müller, K.-R. (2017) Explaining nonlinear classification decisions with deep taylor decomposition. Pattern Recognit 65, 211-222.

(46) Lev, B., Murail, S., Poitevin, F., Cromer, B. A., Baaden, M., Delarue, M., and Allen, T. W. (2017) String method solution of the gating pathways for a pentameric ligand-gated ion channel. Proc. Natl. Acad. Sci. U. S. A. 114, E4158-E4167.

(47) Beauchamp, K. A., Bowman, G. R., Lane, T. J., Maibaum, L., Haque, I. S., and Pande, V. S. (2011) MSMBuilder2: modeling conformational dynamics on the picosecond to millisecond scale. J. Chem. Theory Comput. 7, 3412-3419.

(48) Fleetwood, O. Code to study GPCRs using the String Method with swarms of trajectories. 2019. https://github.com/delemottelab/ gpcr-string-method-2019.

(49) Pan, A. C., Sezer, D., and Roux, B. (2008) Finding transition pathways using the string method with swarms of trajectories. J. Phys. Chem. B 112, 3432-3440.

(50) Fleetwood, O., Kasimova, M. A., Westerlund, A. M., and Delemotte, L. (2019) Molecular insights from conformational ensembles via machine learning. Biophys. J., DOI: 10.1016/ j.bpj.2019.12.016.

(51) Nygaard, R., Zou, Y., Dror, R. O., Mildorf, T. J., Arlow, D. H., Manglik, A., Pan, A. C., Liu, C. W., Fung, J. J., Bokoch, M. P., Thian, F. S., Kobilka, T. S., Shaw, D. E., Mueller, L., Prosser, R. S., and Kobilka, B. K. (2013) The Dynamic Process of $\beta 2$-Adrenergic Receptor Activation. Cell 152, 532-542.

(52) Kofuku, Y., Ueda, T., Okude, J., Shiraishi, Y., Kondo, K., Maeda, M., Tsujishita, H., and Shimada, I. (2012) Efficacy of the $\beta 2$ adrenergic receptor is determined by conformational equilibrium in the transmembrane region. Nat. Commun. 3, 1045-1053-9.

(53) Selent, J., Sanz, F., Pastor, M., and De Fabritiis, G. (2010) Induced Effects of Sodium Ions on Dopaminergic G-Protein Coupled Receptors. PLoS Comput. Biol. 6, e1000884.

(54) Hu, X., Wang, Y., Hunkele, A., Provasi, D., Pasternak, G. W. and Filizola, M. (2019) Kinetic and thermodynamic insights into sodium ion translocation through the $\mu$-opioid receptor from molecular dynamics and machine learning analysis. PLoS Comput. Biol. 15, e1006689.

(55) Selvam, B., Shamsi, Z., and Shukla, D. (2018) Universality of the Sodium Ion Binding Mechanism in Class A G-Protein-Coupled Receptors. Angew. Chem. 130, 3102-3107.

(56) Arnis, S., Fahmy, K., Hofmann, K. P., and Sakmar, T. P. (1994) A conserved carboxylic acid group mediates light-dependent proton uptake and signaling by rhodopsin. J. Biol. Chem. 269, 23879-23881.

(57) McCorvy, J. D., Wacker, D., Wang, S., Agegnehu, B., Liu, J., Lansu, K., Tribo, A. R., Olsen, R. H. J., Che, T., Jin, J., and Roth, B. L. (2018) Structural determinants of 5-HT2B receptor activation and biased agonism. Nat. Struct. Mol. Biol. 25, 787-796.

(58) Wingler, L. M., McMahon, C., Staus, D. P., Lefkowitz, R. J., and Kruse, A. C. (2019) Distinctive Activation Mechanism for Angiotensin Receptor Revealed by a Synthetic Nanobody. Cell 176, 479-490.e12. 
(59) Xu, F., Wu, H., Katritch, V., Han, G. W., Jacobson, K. A., Gao, Z.-G., Cherezov, V., and Stevens, R. C. (2011) Structure of an Agonist-Bound Human A2A Adenosine Receptor. Science 332, 322327.

(60) Wingler, L. M., McMahon, C., Staus, D. P., Lefkowitz, R. J., and Kruse, A. C. (2019) Distinctive Activation Mechanism for Angiotensin Receptor Revealed by a Synthetic Nanobody. Cell 176, 479-490.e12.

(61) Huang, W., Manglik, A., Venkatakrishnan, A. J., Laeremans, T., Feinberg, E. N., Sanborn, A. L., Kato, H. E., Livingston, K. E., Thorsen, T. S., Kling, R. C., Granier, S., Gmeiner, P., Husbands, S. M., Traynor, J. R., Weis, W. I., Steyaert, J., Dror, R. O., and Kobilka, B. K. (2015) Structural insights into $\mu$-opioid receptor activation. Nature $524,315-321$.

(62) Lebon, G., Warne, T., Edwards, P. C., Bennett, K., Langmead, C. J., Leslie, A. G. W., and Tate, C. G. (2011) Agonist-bound adenosine A2A receptor structures reveal common features of GPCR activation. Nature 474, 521-525.

(63) Kruse, A. C., Ring, A. M., Manglik, A., Hu, J., Hu, K., Eitel, K., Hübner, H., Pardon, E., Valant, C., Sexton, P. M., Christopoulos, A., Felder, C. C., Gmeiner, P., Steyaert, J., Weis, W. I., Garcia, K. C., Wess, J., and Kobilka, B. K. (2013) Activation and allosteric modulation of a muscarinic acetylcholine receptor. Nature 504, $101-106$.

(64) Fenalti, G., Giguere, P. M., Katritch, V., Huang, X.-P., Thompson, A. A., Cherezov, V., Roth, B. L., and Stevens, R. C. (2014) Molecular control of $\delta$-opioid receptor signalling. Nature 506, $191-196$.

(65) Eddy, M. T., Lee, M.-Y., Gao, Z.-G., White, K. L., Didenko, T., Horst, R., Audet, M., Stanczak, P., McClary, K. M., Han, G. W., Jacobson, K. A., Stevens, R. C., and Wüthrich, K. (2018) Allosteric Coupling of Drug Binding and Intracellular Signaling in the A2A Adenosine Receptor. Cell 172, 68-80.e12.

(66) Liu, J. J., Horst, R., Katritch, V., Stevens, R. C., and Wuthrich, K. (2012) Biased Signaling Pathways in $\beta 2$-Adrenergic Receptor Characterized by 19F-NMR. Science 335, 1106-1110.

(67) Pietrucci, F. (2017) Strategies for the exploration of free energy landscapes: Unity in diversity and challenges ahead. Rev. Phys. 2, 3245 .

(68) Pan, A. C., and Roux, B. (2008) Building Markov state models along pathways to determine free energies and rates of transitions. $J$. Chem. Phys. 129, 064107-064107-8.

(69) Song, W., Yen, H.-Y., Robinson, C. V., and Sansom, M. S. (2019) State-dependent Lipid Interactions with the A2a Receptor Revealed by MD Simulations Using In Vivo-Mimetic Membranes. Structure 27, 392-403.e3. 